\title{
Intégration des essais randomisés dans les soins cliniques : comment le Canada peut faire mieux
}

\author{
Srinivas Murthy MDCM MScS, Robert A. Fowler MDCM MSc, Andreas Laupacis MD MSc
}

Citation : CMAJ 2020 August 10;192:E928-9. doi : 10.1503/cmaj.201764-f; diffusion hâtive le 30 juillet 2020

Voir la version anglaise de l'article ici : www.cmaj.ca/lookup/doi/10.1503/cmaj.201764

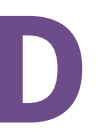

ans un véritable tour de force scientifique et organisationnel, les chercheurs, les soignants et les patients du Royaume-Uni ont rapidement produit des données probantes qui ont transformé les soins aux patients atteints de la maladie à coronavirus 2019 (COVID-19) dans le monde entier. Le Canada devrait suivre leur exemple.

Le 19 mars 2020, l'équipe de l'essai randomisé RECOVERY (pour Randomised Evaluation of COVID-19 Therapy) a recruté son premier patient. L'étude britannique visait notamment à déterminer si l'ajout de la dexaméthasone (un médicament peu dispendieux déjà sur le marché) améliore le traitement habituel des personnes hospitalisées pour cause de COVID-19. Moins de 4 mois et 6424 participants plus tard, les résultats étaient analysés et publiés ${ }^{1,2}$ : la dexaméthasone augmenterait significativement le taux de survie chez les patients dont l'état requiert de l'oxygène d'appoint ou une ventilation mécanique.

Les résultats de l'essai RECOVERY sont si concluants qu'ils ont eu des retombées sur les pratiques partout dans le monde. La randomisation, qui élimine la plupart des facteurs parasites, assure que l'amélioration observée est due à la dexaméthasone et non à des différences dans les caractéristiques des participants. En outre, le principal critère d'évaluation (décès) était sans équivoque; la taille de l'échantillon aléatoire suffisait à la crédibilité des résultats; l'échantillon semblait représentatif de la majorité des personnes atteintes de la COVID-19 (les auteurs estiment avoir recruté environ $15 \%$ des personnes hospitalisées pour cause de COVID-19 au Royaume-Uni3); et les bienfaits de la dexaméthasone étaient considérables (1 patient nécessitant une ventilation mécanique sur 8 survivrait grâce au médicament). Dans d'autres études, l'équipe de RECOVERY a aussi démontré, de manière convaincante, que 2 médicaments très utilisés au début de la pandémie - l'hydroxychloroquine et l'association lopinavir/ritonavir - étaient inefficaces chez les patients hospitalisés atteints de la maladie, ${ }^{4,5}$.

Pour l'instant, le Canada ne peut rivaliser avec le RoyaumeUni dans sa capacité à réaliser des études de première importance. Mais que pouvons-nous apprendre des exploits britanniques?
Le Royaume-Uni avait déjà, intégré à son service de santé national (le National Health Service, ou NHS), un réseau de recherche clinique et d'amélioration des soins financé à long terme pour mener les projets de recherche approuvés par le National Institute for Health Research (NIHR) dans un processus d'évaluation par les pairs ${ }^{6}$. Les centres participants reçoivent du soutien à l'infrastructure pour exécuter leurs travaux, et peuvent, en de rares occasions, changer leur fusil d'épaule en bloc pour se concentrer sur un même projet. Les médecins hygiénistes en chef du pays et le NIHR ayant statué que l'essai RECOVERY était prioritaire ${ }^{7,8}, 176$ organisations hospitalières universitaires et non universitaires britanniques s'y sont joints ${ }^{2}$. Le taux de participation, remarquablement élevé, accroît le caractère généralisable des résultats. II montre aussi que, pour les professionnels de la santé, la question de recherche était importante et la participation à l'essai ne compliquait pas indûment les soins. La collecte de données locale se limitait aux renseignements essentiels; les résultats des patients provenaient d'une base de données administratives centralisée, dont le contenu était accessible aux chercheurs (moyennant un consentement, dans le respect des mesures de protection des renseignements personnels pertinentes) pratiquement en temps réel. D'autres facteurs expliquent le succès de l'entreprise britannique, entre autres la rapidité et la centralité - à l'échelle nationale - de l'évaluation éthique, et la possibilité qu'ont les cliniciens d'expliquer aux patients les implications de leur participation à l'essai (ici, les soins cliniques et le consentement à participer à un essai clinique sont 2 éléments séparés).

L'essai RECOVERY fait la preuve qu'il est possible de boucler un essai clinique de grande envergure bien conçu et exécuté, lorsqu'il est jugé prioritaire, en 4 mois seulement. En contexte canadien, cette façon de faire pourrait servir dans beaucoup de dossiers de santé importants autres que la COVID-19. Prenons l'exemple des maladies non transmissibles lourdes de conséquences pour les patients et lourdes en ressources pour les services de santé et les services sociaux du pays : elles seraient bien mieux prises en charge si nous les examinions dans des essais de première importance intégrés aux soins habituels. Les Instituts de recherche en santé du Canada (IRSC) 
ont certes investi dans la recherche sur la COVID-19 ${ }^{9}$ et d'autres essais, mais les chercheurs et les patients motivés restent freinés par une capacité de recherche clinique déficiente et l'intégration insuffisante, en temps réel, de données pancanadiennes dans les soins.

Ceux qui gèrent et financent les systèmes de santé au Canada doivent considérer les essais de première importance comme inhérents aux soins cliniques et assurer un financement suffisant et stable des infrastructures. Ainsi, nous n'aurions plus à passer par le long et coûteux processus d'établissement d'ententes et de contrats de partage de données distincts avec de multiples hôpitaux et organisations. Même si la collaboration entre les provinces et les territoires est plus difficile qu'au sein du NHS, elle serait possible avec la contribution financière et l'aide du fédéral. Il faudrait mettre en place, vraisemblablement par l'intermédiaire des IRSC, un mécanisme d'évaluation des retombées cliniques et systémiques potentielles et de la rigueur méthodologique des essais cliniques qui servirait à déterminer ceux qui sont des priorités nationales. Ce mécanisme commanderait la formation d'un comité d'examen national composé de scientifiques, de professionnels de la santé, de patients et de gestionnaires du réseau de la santé. Les essais cliniques prioritaires seront efficients, utiliseront des critères d'inclusion simples et rassembleront des données pour des critères d'évaluation importants, mais limités, notamment des données cliniques à collecte systématique diffusées entre les provinces et rapidement accessibles (plus rapidement que les quelques mois à 1 an présentement nécessaires pour obtenir des données administratives à des fins de recherche).

En conclusion, les Canadiens atteints de la COVID-19 profitent de la prévoyance du Royaume-Uni. Pour que des essais comme RECOVERY aient lieu ici, il faudrait un changement de culture majeur à l'égard des essais cliniques, et aussi une volonté politique, du financement et des infrastructures. C'est chose possible, et il faut faire mieux.

\section{Références}

1. Horby P, Lim WS, Emberson J, et al.; RECOVERY Collaborative Group. Effect of dexamethasone in hospitalized patients with COVID-19: preliminary report. medRxiv 22 juin 2020. doi: 10.1101/2020.06.22.20137273.

2. RECOVERY Collaborative Group; Horby P, Lim WS, Emberson JR, et al. Dexamethasone in hospitalized patients with COVID-19 - preliminary report. New Engl J Med 17 juillet 2020. [Cyberpublication avant impression]. doi: 10.1056/ NEJMoa2021436.
3. Nuffield Department of Population Health. No clinical benefit from use of hydroxychloroquine in hospitalised patients with COVID-19: Statement from the Chief Investigators of the Randomised Evaluation of COVid-19 thERapY (RECOVERY) Trial on hydroxychloroquine, 5 June 2020. Oxford (Royaume-Uni); RECOVERY - Randomised Evaluation of COVID-19 Therapy. 2020. Accessible ici : www.recoverytrial.net/news/ statement-from-the-chief-investigators-of-the-randomised-evaluation -of-covid-19-therapy-recovery-trial-on-hydroxychloroquine-5-june-2020 -no-clinical-benefit-from-use-of-hydroxychloroquine-in-hospitalised -patients-with-covid-19 (consulté le 20 juillet 2020).

4. Nuffield Department of Population Health. No clinical benefit from use of lopinavir-ritonavir in hospitalised COVID-19 patients studied in RECOVERY. Oxford (Royaume-Uni); RECOVERY - Randomised Evaluation of COVID-19 Therapy. 2020. Accessible ici : www.recoverytrial.net/news/no-clinical-benefit -from-use-of-lopinavir-ritonavir-in-hospitalised-covid-19-patients-studied-in -recovery (consulté le 20 juillet 2020).

5. Kupferschmidt K. One U.K. trial is transforming COVID-19 treatment. Why haven't others delivered more results? [En ligne] Science 2 juillet 2020.

6. National Institute for Health Research. Clinical Research Network. Londres (Royaume-Uni); [En ligne]. Accessible ici : www.nihr.ac.uk/explore-nihr/support/ clinical-research-network.htm (consulté le 15 juillet 2020).

7. Atherton F, Calderwood C, McBride M, et al. Novel coronavirus: Clinical trials [lettre]. Oxford (Royaume-Uni); RECOVERY - Randomised Evaluation of COVID19 Therapy, Nuffield Department of Population Health. 2020. Accessible ici : www.recoverytrial.net/files/professional-downloads/the-importance-of-covid -19-clinical-trials.pdf (consulté le 15 juillet 2020).

8. Whitty C, Powis S. Randomised evaluation of COVID-19 therapy (RECOVERY trial) [lettre]. Oxford (Royaume-Uni); RECOVERY - Randomised Evaluation of COVID-19 Therapy, Nuffield Department of Population Health. 16 mars 2020. Accessible ici : www.recoverytrial.net/files/recovery-trial-cmo-and-nhse-md. pdf (consulté le 15 juillet 2020).

9. 2020 funding decision notifications. Ottawa: Canadian Institutes of Health Research; modified 2020 July 16.. Accessible ici : https://cihr-irsc.gc.ca/f/196. html (consulté le 15 juillet 2020).

Intérêts concurrents : Voir www.cmaj.ca/site/misc/cmaj_staff.xhtml pour Andreas Laupacis. Aucun autre intérêt concurrent déclaré.

Affiliations : Département de pédiatrie (Murthy), Université de la Colombie-Britannique, Vancouver (C.-B.); Division interdépartementale des soins intensifs (Fowler), Département de médecine et Institut des politiques, de la gestion et de l'évaluation de la santé (Fowler), Université de Toronto; Centre des sciences de la santé Sunnybrook (Fowler), Toronto (Ont.); rédacteur en chef (Laupacis), CMAJ.

Correspondance : Rédaction du CMAJ, editorial@cmaj.ca 